\title{
SAT-COMM - FADE INTEFERANCE AND BAND MITIGATION ANALYSIS
}

\author{
Ravi Kumar*1, Ritika Ghai ${ }^{2}$ \\ *1(raviy516@gmail.com), ${ }^{2}$ (ritkaghai7@gmail.com)
}

*Corresponding Author: -

Email: raviy516@gmail.com

\section{Abstract: -}

When it comes to address the capacity issue in satellite communication, the first technique to consider is the multi-beam coverage, with a high number of beams that allow a high degree of frequency reuse. In order to increase even more that capacity, the second step is to utilize higher frequency bands such as $\mathrm{Ka}(20-30 \mathrm{GHz}), \mathrm{Q} / \mathrm{V}(40-50 \mathrm{GHz})$ or EHF (20-45 $\mathrm{GHz}$ ) bands, where respectively, $1 \mathrm{GHz}, 3 \mathrm{GHz}$ and $2 \mathrm{GHz}$ are allocated to the Fixed Satellite Service (FSS). The first commercial satellites with Ka-band transponders are today in operation, and it is expected that the congestion in lower frequency bands like Ku-band will push new systems into moving progressively to Ka band and, in a longer term, to $Q / V$ $b$ and. Depending on the type of mission, Ka-Q/V band satellite could be envisaged as, for example, for two way broadba nd access services characterised by a high asymmetrical traffic, using part of the Ka-band for user access while data distribution service could take most advantage of wider bandwidth in $Q / V$ band.

Although $\mathrm{Ka}$ and $\mathrm{Q} / \mathrm{V}$ bands are attractive from the point of view of the amount of frequency bandwidth that the satellite can potentially use, some important limitation could moderate the enthusiasm of using them if specific techniques were not implemented in the satellite system to guarantee the capacity, the availability and the quality of service. The major limitation is the effect of radio-wave propagation through the lowest layers of the atmosphere. As the operating frequenc $y$ is increased, the attenuation and scintillation effects of atmospheric gas, clouds and rain become more severe, the direct consequence is the need to implement high system static margins, in order to insure a minimum outage duration of the service, for a given objective of link availability. However, technology limitation (on both terrestrial and space segments) combined with cost efficiency requirements refrain from considering fixed static margins as the only mean to compens ate propagation impairments at high frequency bands, and push towards the implementation of Fade Mitigation Techniques (FMT). The aim of this paper is to present the main issues in designing FMTs for Ka-Q/V band satellite communication systems. First of all, the conventional design of the physical layer of a satcom system is outlined. Afterwards, a revi ew of FMT concepts and related interference issues are presented followed by the examination of the impact on upper la yer. Finally, the main aspects of FMT design are investigated before proposing some recommendations. IFMT practical implementation (detection scheme and estimation techniques, consequences on signalling and impact on synchronisation) will not be dealt with in this paper as it is the object of [3].

\section{(a) $(\$)$}




\section{PROPAGATION CHANNEL INFLUENCE}

The methodology followed to design the physical layer of satellite communication systems, especially at Ku-band, relies on the implementation of a propagation margin to take into account for rain attenuation. The link budgets are actually closed by solving a worst-case analysis. The worst case is selected for a specified percentage of the total coverage (between $90 \%$ and $95 \%$ ), on the one hand with respect to attenuation and on the other hand with respect to carrier-tointerference ratio. It corresponds generally to both edge of coverage and low elevation.

Then, a performance assessment is performed for the worst-case location to obtain in a first step the corresponding avail ability (or outage time), and in a second step to evaluate system capacity or throughput. Figure 1 shows predicted CDF o $\mathrm{f}$ total impairment performed with Recommendation ITU-R P.618, between a GEO satellite $\left(11^{\circ} \mathrm{W}\right)$ and the town of Bar $\mathrm{i}$ (South Italy) which constitutes a worst case in Europe with respect to attenuation.
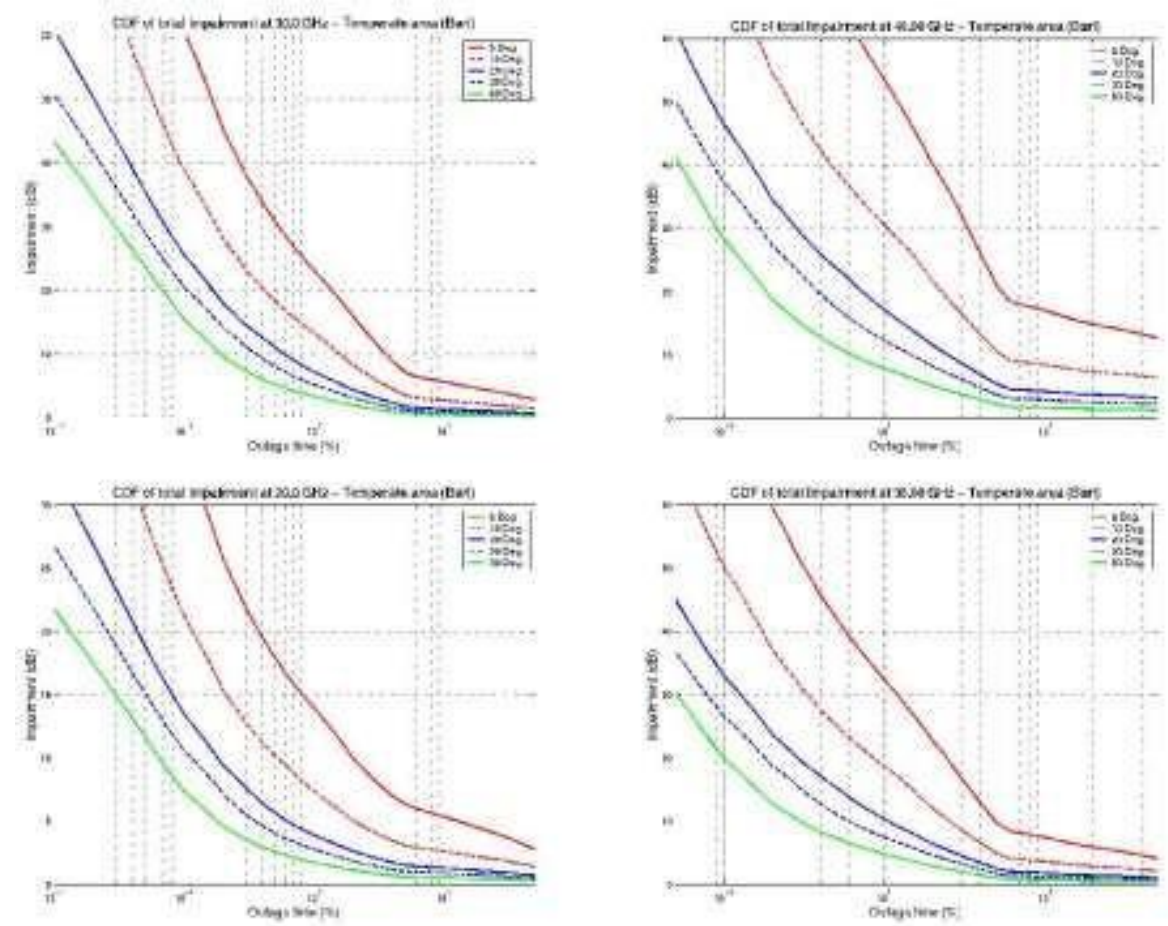

Figure 1: Example of predictions of total impairments at Ka-band uplink (upper left) and downlink (lower left) and at $Q / V$ band uplink (upper right) and downlink (lower right)

Assuming that Ka-band is going to be used for consumer or corporate markets [4], a target availability between $99.5 \%$ and $99.9 \%$ could be required for these systems. Therefore, static margins of $10 \mathrm{~dB}$ on the uplink and of $6 \mathrm{~dB}$ on the downlink may allow the $99.5 \%$ availability requirement to be fulfilled at Ka-band for a European coverage. In this case, the main issue regarding FMTs is: how to increase system throughput?

As far as $\mathrm{Q} / \mathrm{V}$ band is concerned, it may be used in the future either for star network feeder links or for backbone applications [4]. In both cases, a target availability between $99.95 \%$ and $99.99 \%$ of the time could be required. For these tim e percentages, in a conventional system design, several tens of dBs would have to be compensated with a static margin, which is not possible due to techology limitations, leading to low availability only, even for a European coverage. Therefore, FMTs will have the following objectives at Q/V bands: on the one hand, how to improve system availability? On the other hand, how to increase system throughput?

\section{REVIEW OF FADE MITIGATION TECHNIQUES}

Making use of Fade Mitigation Techniques involves adapting in real time the link budget to the propagation conditions through some specific parameters such as power, data rate, coding etc. However, this real time adaptivity has an impact not only on carrier-to-noise ratios but also on carrier-to-interference ratios and on upper layers. Both aspects have therefore to be carefully studied.

\subsection{FMT concepts}

Various methods exist to counteract propagation effects at the physical layer level. The most relevant ones should take into account operating frequency bands, performance objectives of the system and geometry of the network (system arch itecture, multiple access schemes, ...). A lot of publications have been written up to now on the subject [5-9] and a review of FMTs has been realised in the framework of COST 255 [2]. In fact FMT for the physical layer can be divided into :

- Power Control: transmitting power level fitted to propagation impairments,

- Adaptive waveform: fade compensated by a more efficient modulation and coding scheme,

- Diversity: fade avoided by the use of another less impaired link, 
- Layer 2 : coping with the temporal dynamics of the fade.

Power Control, adaptive waveform and layer 2 FMTs are taking advantage of unused in-excess resource of the system, whereas diversity FMT implies a re-route strategy. Techniques sharing unused resource aim to compensate fading occur ring on a given link in order to maintain or to improve the link performance (required $\mathrm{C} / \mathrm{N}_{0}$ ). Diversity techniques maintain link performance by changing the frequency band or the link geometry.

\subsubsection{Power control}

Four types of Power Control FMT can be considered: Up-Link Power Control (ULPC), End-to-End Power Control (EE PC), Down-Link Power Control (DLPC) and On-Board Beam Shaping (OBBS).

The aim of ULPC, the output power of a transmitting Earth station is matched to uplink impairments. Transmitter power is increased to counteract fade or decreased when more favourable propagation conditions are recovered so as to limit interference in clear sky conditions and therefore to optimise satellite capacity. In the case of transparent payloads, ULP $\mathrm{C}$ can prevent from reductions of satellite EIRP caused by the decreased uplink power level that would occur in the absence of ULPC.

EEPC can be used for transparent configuration only. Indeed, the output power of a transmitting Earth station is matched to up-link or down-link impairments. In the case of regenerative repeaters, up and down links budgets are independent, so the concept of EEPC cannot exist anymore. EEPC is used to keep a constant overall margin of the system. As for U LPC, transmitter power is increased to counteract fade or decreased when more favourable propagation conditions are recovered to limit interference and optimise satellite capacity.

With DLPC, the on-board channel output power is adjusted to the magnitude of downlink attenuation. DLPC aims to all ocate a limited extra-power on-board in order to compensate a possible degradation in term of down-link $\mathrm{C} / \mathrm{N}_{0}$ due to propagation conditions on a particular region. In this case, all Earth stations in the same spot beam benefit from the improvement of EIRP.

OBBS technique is based on active antennas, which allows spot beam gains to be adapted to propagation conditions. Actually, the objective is to radiate extra-power, and to compensate rain attenuation only on spot beams where rain is likel y to occur.

\subsubsection{Adaptive waveform}

These FMTs could be split into Adaptive Coding (AC), Adaptive Modulation (AM) and Data Rate Reduction (DRR).

The introduction of redundant bits to the information bits when a link is experiencing fading, allows detection and correction of errors $(\mathrm{FEC}, \ldots$ ) caused by propagation impairments and leads to a reduction of the required energy per information bit. Adaptive coding consists in implementing a variable coding rate matched to impairments originating fr om propagation conditions.

Higher system capacity for a given bandwidth can be achieved with spectral efficient modulation schemes but in clear $\mathrm{s}$ ky conditions only due to link budget power limitation. As Adaptive Coding, the aim of Adaptive Modulation is to decrease the required energy per information bit required corresponding to a given BER, which translates into a reduction of

the spectral efficiency as $\mathrm{C} / \mathrm{N}_{0}$ decreases. The reduction of the spectral efficiency is the results of the use of lower-level modulation schemes.

Further reduction can be obtained by a decrease of the information data rate at constant BER. The technique is called Data Rate Reduction. Here, user data rates should be matched to propagation conditions: nominal data rates are used under clear sky conditions (no degradation of the service quality with respect to the system margin), whereas reductions are introduced according to fade levels.

Differently from ULPC which aims at restoring the Carrier-to-Noise Ratio $\left(\mathrm{C} / \mathrm{N}_{0}\right)$ through an increase of the Earth Station transmitted power, Adaptive Coding and Modulation as well as Data Rate Reduction allow a decrease in the required $\mathrm{C} / \mathrm{N}_{0}$ while maintaining the link performance in term of BER. AC and AM aim at a reduction in the required energy-perinformation bit level $\left(\mathrm{E}_{\mathrm{b}} / \mathrm{N}_{0}\right)$, which translates into a decrease in the required $\mathrm{C} / \mathrm{N}_{0}$.

\subsubsection{Diversity}

The objective of these techniques is to re-route information in the network in order to avoid impairments due to an atmospheric perturbation. Three types of diversity techniques can be considered: site (SD), satellite (S at D) and frequency (F D) diversity. These techniques are very expensive as the associated equipments have to be redundant.

SD is based on the change of the network routes; therefore, it applies only for the Fixed Satellite Service. SD takes 
advantage of the fact that two fades experienced by two Earth Stations separated by a distance higher than the size of a convective rain cell (at least $10 \mathrm{~km}$ ), are statistically independent. The Earth station affected by a weaker event is used and th e information is routed to the original destination through a separated terrestrial network.

Satellite Diversity can be regarded in two different ways: on one hand, when designing the system, by optimising the size of the constellation (that is the number of satellites) in order to prevent communications at low elevation angles. On t he other hand in allowing Earth Stations to choose between various satellites, the one for which the most favourable link with respect to the propagation conditions exists.

Frequency Diversity is a technique based on the fact that payloads using two different frequency bands are available on board. When a fade is occurring, links are re-routed using the lowest frequency band payload, less sensitive to atmospheric propagation impairments.

\subsubsection{Layer 2}

FMT at layer 2 level are techniques which do not aim at mitigating a fade event but instead rely on the re-transmission o $\mathrm{f}$ the message. Two different techniques can be envisaged at layer 2 : Automatic Repeat Request (ARQ) and Time Diversity (TD). With ARQ, the message is sent regularly untill the message reaches successfully the receiver. ARQ with a random or predefined time repetition protocol would be an alternate solution.

Time diversity can be considered as a FMT that aims to re-send the information when the state of the propagation channel allows to get through. In this case, most often, there is no need to receive the data file in real time and it is acceptable for the user point of view to wait for the end of the propagation event (in general some tens of minutes) or for a decreas e of traffic. This technique benefits from the use of propagation mid-term prediction model in order to estimate the most appropriate time to re-sent the message without repeating the request.

\subsubsection{Joint FMTs}

Previous works performed in the frameworks of COST 235 [10] and COST 255 [2] pointed out two important aspects $\mathrm{f}$ or new systems operating at $\mathrm{Ka}$ and $\mathrm{Q} / \mathrm{V}$-bands:

- On the one hand, it appears that each FMT is more or less adapted to a specific range of availability. Then these fade mitigation methods are quite complementary and can be implemented simultaneously (joint FMTs) to extend the availability range of requirements.

- On the other hand, such methods implemented individually can only relatively compensate small propagation impairments. It will be possible to regularly improve the performance of the mitigation to a large extent, by carrying out a combination of different kinds of FMTs when there is a need to transmit high priority information.

\section{INTERFERENCE ISSUES}

The objective of this section is to investigate the impact of FMTs on interference level. To carry out this investigation, interference sources are first of al reviewed for both up and down links.

\subsection{Interference sources on the uplink}

Four types of interference can be identified for the uplink : adjacent channel interference, co-channel interference and cross-channel interference and adjacent system interference (see Figure 2). These interference are produced at the input of the satellite transponder, by carriers transmitted by Earth stations either belonging to the same system or to another system. In an ideal system these carriers should be strongly attenuated, due to access scheme, frequency and polarisation plan, antenna pattern and filtering issues.
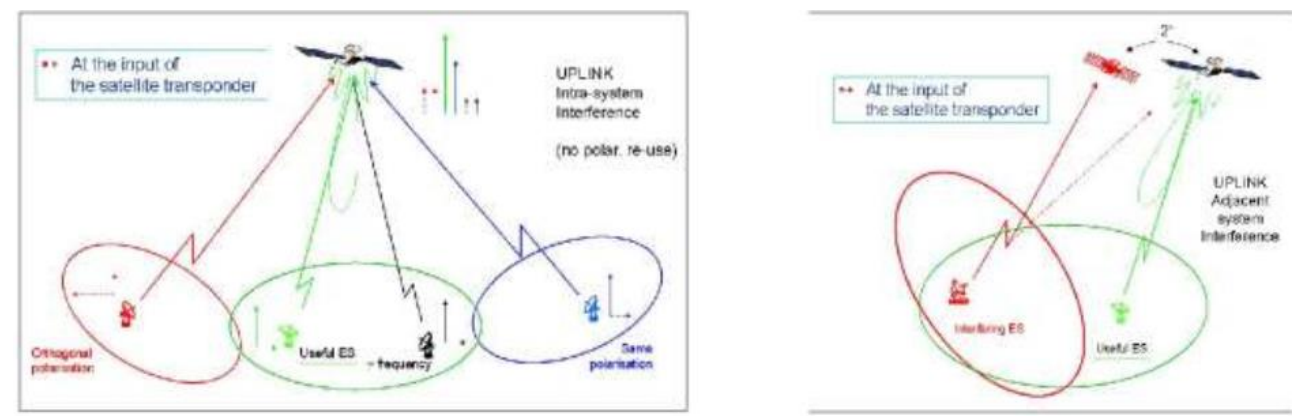

Figure 2: Uplink interference (no polarisation re-se)

Adjacent channel interference is produced by carriers belonging to Earth stations of the same system, located in the same spot beam as the useful Earth station, transmitted at a different frequency and at the same polarisation. In FDMA and TDMA access schemes, these carriers interfere with the useful carrier because of the non-ideal performance of the transmit Earth station filter. 
Co-channel interference is produced by carriers belonging to Earth stations of the same system, transmitted the same frequency and at the same polarisation as the useful Earth station carrier. These interfering Earth stations are located in a different spot beam as the useful Earth station in FDMA and TDMA, but they are located in the same spot beam as the useful Earth station in CDMA. In FDMA and TDMA, this interference is conditioned by the satellite antenna pattern in th e direction of the interferer whereas in CDMA, it is limited by code correlation properties.

Cross-channel interference is produced by carriers belonging to Earth stations of the same system, transmitted at the sa me frequency and at the orthogonal polarisation as the useful Earth station carrier. These interfering Earth stations are located in a different spot beam as the useful Earth station if single polarisation is used, but they are located in the same $\mathrm{s}$ pot beam as the useful Earth station for dual polarisation systems. For single polarisation systems, this interference is limited by the satellite antenna roll-of in the direction of the interferer and by the isolation in polarisation of the satellite antenna, whereas in the case of polarisation re-use, it is limited by the polarisation isolation of satellite and Earth station antennas only.

Adjacent system interference is produced by carriers belonging to Earth stations of another satcom system, transmitted a $t$ the same frequency and polarisation as the useful Earth station carrier. This interference is limited by the angular separation of the two satellites from the interfering Earth station position (see Figure 2). For the uplink, interference fro m terrestrial systems (Broadband Fixed Wireless Access or radar systems) can be considered as negligible apart from specific cases such as very low elevation links.

\subsection{Interference sources on the downlink}

Similarly, four types of interference can be identified for the downlink : adjacent channel interference, co-channel interference and cross-channel interference and adjacent system interference (see Figure 3). These interference are produced at the input of the received Earth station, by carriers transmitted by either the satellite of the considered system or by a satellite of another system. In an ideal system these carriers should be strongly attenuated, due to multiplex scheme, frequency and polarisation plan, antenna pattern and filtering issues.

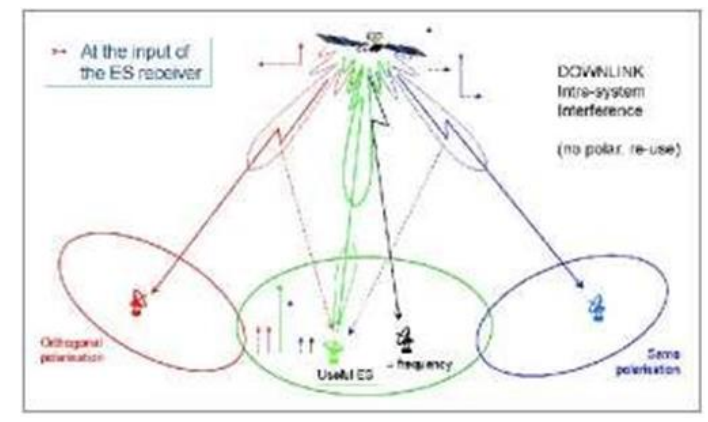

Figure 3: Downlink interference (no polarisation re-use)

Adjacent channel interference is produced by carriers transmitted by the satellite to Earth stations of the same system, located in the same spot beam as the useful Earth station, transmitted at a different frequency and at the same polarisation. In FDMA and TDMA access schemes, these carriers interfere with the useful carrier because of the non-ideal performance of the received Earth station filter.

Co-channel interference is produced by carriers transmitted by the satellite to Earth stations of the same system, at the same frequency and at the same polarisation as the useful carrier. These interfering carriers are sent to Earth stations located in a different spot beam as the useful Earth station in FDMA and TDMA, but located in the same spot beam as the useful Earth station in CDMA. In FDMA and TDMA, this interference is limited by the satellite antenna roll-of of the adjacent spot beam in the direction of the useful Earth station whereas in CDMA, it is limited by code correlation properties.

Cross-channel interference is produced by carriers transmitted by the satellite to Earth stations of the same system, at th e same frequency and at the orthogonal polarisation as the useful carrier. These interfering carriers are sent to Earth stations located in a different spot beam as the useful Earth station if single polarisation is used, but to Earth stations located in the same spot beam as the useful Earth station for dual polarisation systems. For single polarisation systems, this inte rference is limited by the satellite antenna roll-of of the adjacent spot beam in the direction of the useful Earth station an $\mathrm{d}$ by the isolation in polarisation of the satellite antenna. In the case of polarisation re-use, cross-channel interference is limited by the polarisation isolation of both satellite and Earth station antennas only.

Adjacent system interference is produced by carriers transmitted by another satellite to Earth stations of another satcom system, transmitted at the same frequency and polarisation as the useful carrier. This interference is limited by the angular separation of the two satellites from the useful Earth station position (see Figure 3). For the downlink, interference from terrestrial systems (Broadband Fixed Wireless Access or radar systems) have to be evaluated, especially for low elevation 
links.

Remark: Even if it cannot be strictly considered as interference, multicarrier effects (intermodulation noise, carrier supression) can be considered as a fifth source in the downlink interference budget.

Multicarrier effects are produced by satellite power amplifiers operated at or close to saturation. In this configuration, due to non-linearity, intermodulation products are generated and the satellite output power is shared between the useful carriers and the intermodulation noise. On the other hand, carriers having a different power are amplified in a differen $t$ way, leading to suppression of weak carriers by strong ones.

\subsection{Preliminary analysis of the impact of FMT on interference}

In link budget calculations, interference is taken into account through the $\mathrm{C} / \mathrm{I}_{0}$, which is the ratio of the useful carrier power to the interfering carrier power. Therefore, to study the impact of FMTs on interference, it is natural to consider on t he one hand power control techniques and on the other hand the other FMTs.

\subsubsection{Power Control:}

In satellite communication systems, ULPC allows two different objectives to be reached: static compensation of interference and dynamic fade mitigation. The aim of the static power control (SPC) is to keep a constant level of all the carrier s at the input of the satellite transponder in clear sky conditions to limit non-linear effects such as intermodulation noise or carrier suppression. The objective is actually to compensate for satellite antenna roll-off and mispointing, free space loss and long-term drifts of RF chains [11]. With dynamic ULPC, the output power of a transmitting Earth station is matched to uplink impairments. Therefore, ULPC can be used to keep a constant level of all the carriers at the input of the transponder, as for static power control but both in clear sky and in rain conditions, while maintaining the uplink budget $\mathrm{c}$ lose to target. With ULPC, transmitter power is increased to counteract fade or decreased when more favourable propagation conditions are recovered; ULPC is therefore a valuable technique to maintain the interference at the same level as in clear sky conditions.

In transparent systems only, EEPC can be used to maintain the overall link budget but in this case the carrier power level at the input of the transponder can vary. Therefore, uplink and downlink $\mathrm{C} / \mathrm{N}_{0}$ are modified all together with the corresponding $\mathrm{C} / \mathrm{IM}_{0}$ and $\mathrm{C} / \mathrm{I}_{0}$.

With EIRP control techniques, Down-Link Power Control (DLPC) and On-Board Beam Shaping (OBBS), the power on the downlink is distributed in a different way to mitigate propagation impairments. However, if the decision is taken to increase satellite EIRP to mitigate propagation impairments occurring in a given spot beam (even packet by packet), it w ill cause higher interference (co-channel and cross-channel) on other spot beams and the resulting downlink $\mathrm{C} / \mathrm{I}$ increase s mechanically. On the other hand, DLPC triggering results in an increase of the transponder operation point towards saturation and consequently in an increase of intermodulation noise. The activation of DLPC or OBBS leads therefore to a higher interference contribution to the link budget.

\subsubsection{Other FMTs:}

With Adaptive Wave-Form FMTs, there is no direct action on the $\mathrm{C} / \mathrm{N}_{0}$, but instead on the required $\mathrm{E}_{\mathrm{b}} / \mathrm{N}_{0}$ or on the data rate. Consequently, if the coding, the modulation scheme or the data rate is matched to the propagation conditions, the carrier power at the input of the satellite will go on decreasing according to propagation impairments. If on the other hand interfering Earth stations remain in clear sky conditions, the C/I ratio will increase for the attenuated carrier and will de crease for the other, if Adaptive Coding (AC) or Adaptive Modulation (AM) is applied at constant bandwidth). Moreove $\mathrm{r}$, for the uplink, the decrease in carrier power at the input of the satellite could lead to carrier supressions. If AC or AM is implemented at variable bandwidth, not only the $\mathrm{C} / \mathrm{I}_{0}$ but also directly the interference level density $\mathrm{I}_{0}$ will vary as the power might be distributed on a smaller bandwidth.

With diversity techniques, the action of the FMT is not to modify the link budget but instead to establish a new one. The impact of diversity on interference will therefore depend on the considered scenario. For instance, if Site Diversity (SD) is applied with two Earth stations operated continuously at the same time or switched, when necessary, the consequences on interference will not be the same. Similarly, for Frequency Diversity (FD), the impact will be strongly dependent on the utilization strategy of the lower frequency band payload (back-up or used for traffic). In the conventional application of SD (switched gateways) and FD (back-up payload only), there is no impact of FMT on interference. Similarly, with layer two re-transmission techniques (ARQ or time diversity), the physical layer is not modified by the FMT activation, therefore, there is no impact of these FMTs on interference.

\section{CONCLUSION}

The objective of this paper has been to present a review of Fade Mitigation Techniques for satellite communication systems operating at Ka and Q/V bands. Four types of FMTs have been identified in this paper: power control, adaptive waveform, diversity and layer 2 FMTs. Although different in their principles, these FMTs are complementary and combine d use of different FMTs is required when high impairments have to be mitigated, first of all to improve system 
availability, secondly to limit interference and thirdly to increase system capacity.

Interference issue has been analysed in a second step. A review of the different sources of interference has been presente $\mathrm{d}$ for up and down links, including adjacent channel, co-channel, cross-channel and adjacent system interference. Multicarrier effects such as intermodulation noise and carrier suppression have been considered too. Finally, the impact of FM Ts on interference has been briefly analyzed. It appears that some of these techniques have a strong impact on interference if they act directly on the link budget through: an adjustment of the power (power control techniques), no adjustment of the carrier-to-noise ratio or an adjustment of the carrier bandwidth (adaptive waveform techniques). For diversity, no clear impact on interference has been put into evidence as it is strongly dependent on the considered scenario. Finally, $r$ e-transmission techniques do not seem having any impact on interference.

This work has been focused essentially on FMT concepts and on interference issues. However, FMT definition clearly impacts synchronisation, signalling and upper layers, in particularly the MAC layer through the resource management [1 2]. On the other hand, FMT design cannot be carried out without considering their practical implementation, which requires to define the fade detection scheme, the estimation technique and the control logic [13]. Careful analysis and simulation have to be carried out in order to optimise a FMT control loop and to assess physical layer performances [14]. Advanced short-term prediction techniques improve the performance of a FMT control loop [15].

\section{REFERENCES}

[1]. Salonen E. et al. : "Modelling and calculation of atmospheric attenuation for low-fade-margin satellite communications", ESA Journal, Vol. 16, n 3, 1992, pp. 299-317.

[2]. COST 255 : "Radiowave propagation modelling for new satcom services at Ku-band and above", COST 255 Final Report, Chapter 5.3, ESA Publications Division, SP-1252, March 2002.

[3]. Grémont B. : "Event detection, control and performance modelling", COST 272-280 Workshop, Noordwijk, The Netherlands, May 2003, this issue.

[4]. Arbesser-Rastburg B., Bellini M., Coromina F., De Gaudenzi R., Del Rio-Herrero O., Hollreiser M., Rinaldo R., Rinous P., Roederer A. : "R\&D Directions for next generation broadband multimedia systems : an ESA perspective", AIAA Conference, Montreal, Canada, May 2002.

[5]. Willis MJ., Evans BG. : "Fade countermeasures at Ka-band for OLYMPUS", Int. Jour. Sat. Com., Vol. 6, June 88, pp. 301-311.

[6]. Tartara G. : "Fade countermeasures in millimetre-wave satellite communications : a survey of methods and problems", Proc. Olympus Util. Conference, Vienna, Austria, April 1989.

[7]. Gallois A.P. : "Fade countermeasure techniques for satellite communication links", Int. Symp. on Comms Theory and Applications, July 1993.

[8]. Acosta R.J. : "Rain fade compensation alternatives for Ka-band communication satellites", 3rd Ka-band Utilization Conference, Sorrento, Italy, 15-18 Sept. 1997.

[9]. Castanet L. - Lemorton J. - Bousquet M. : "Fade Mitigation techniques for New SatCom services at Ku-band and above : a Review", Fourth Ka-band Utilization Conference, Venice, 2-4 November 1998.

[10].COST 235 : "Radiowave propagation effects on next generation fixed-services terrestrial telecommunications systems", Final report, n EUR 16992 EN, Chapter 3, pp. 159-168, 1996.

[11].Dodel H. - Riedl C. : "Can ULPC double satellite capacity ?", 2nd Euro. Conf. on Sat. Com., Liège, Belgium, 22-24 Oct.1991.

[12].Pech P., Castanet L., Radzik J., Bousquet M. : "Insights into an architecture of Ka-band OBP satellite system involving a Fade Mitigation Technique (FMT) : challenges, simulation and performance", $8^{\text {th }}$ Ka-band Utilization Conference, Baveno, Italy, 2527 September 2002.

[13].Castanet L. : "Fade Mitigation Techniques for new SatCom systems operating at Ka and V bands", Ph'D of SUPAERO, Toulouse, France, December 2001.

[14].Castanet L., Mertens D., Bousquet M. : "Simulation of the performance of a Ka-band VSAT videoconferencing system with uplink power control and data rate reduction to mitigate atmospheric propagation effects", Int. J. Satell. Commun., Vol. 20, n⿳⺈, JulyAugust 2002, pp. 231-249.

[15].Bolea-Alamañac A., Bousquet M., Castanet L., Van de Kamp M.M.J.L. : "Impact of short-term prediction models in Fade Mitigation Techniques control loops", COST 272-280 Workshop, Noordwijk, The Netherlands, May 2003, this issue. 